\title{
Treatment adherence in multiple sclerosis: a survey of Belgian neurologists
}

\author{
This article was published in the following Dove Press journal: \\ Patient Preference and Adherence \\ 19 November 2015 \\ Number of times this article has been viewed
}

\section{Danny Decoo' \\ Mathieu Vokaer ${ }^{2}$ \\ 'Department of Neurology and Neurorehab, AZ Alma, Sijsele, Belgium; 2Multiple Sclerosis Clinic, Edith Cavell Hospital, CHIREC group, Brussels, Belgium}

Background: Poor treatment adherence is common among patients with multiple sclerosis (MS) This survey evaluated neurologists' perception of treatment adherence among MS patients.

Materials and methods: This questionnaire-based survey of Belgian neurologists treating MS patients was conducted between June and July 2014. Face-to-face interviews with the neurologists were based on a semistructured questionnaire containing questions regarding the perception of the treatment-adherence level.

Results: A total of 41 neurologists participated in the survey. Of these, $88 \%$ indicated frequent discussions about treatment adherence as beneficial for treatment efficacy. The mean time spent on the treatment-adherence discussion during the initial consultation was 11 minutes, with $24 \%$ of doctors spending 5 minutes and $24 \%$ of doctors spending 10 minutes discussing this issue. The majority of neurologists (56\%) perceived the adherence level in MS as good, and $12 \%$ perceived it as excellent. The majority of neurologists (64\%) indicated intolerance as a main cause of poor adherence, and all neurologists reported insufficient efficacy as a consequence of nonadherence. The importance of adherence in the neurologists' practice was evaluated on a scale of $1-10$, with $1=$ "not very important" and $10=$ "very important": $44 \%$ of doctors indicated a score of 10, and the mean score was 9.0.

Conclusion: Belgian neurologists consider treatment adherence in MS as essential for the benefits of therapies. However, although neurologists are aware of the consequences of nonadherence, they generally spend limited time discussing the importance of treatment adherence with their patients.

Keywords: multiple sclerosis, treatment adherence, physician survey

\section{Introduction}

Treatment adherence, defined as a patient's acceptance of the need for a medication, persistence with the therapy, and compliance, is crucial for achieving optimal clinical outcomes. ${ }^{1}$ Nonadherence of patients with chronic illnesses to long-term therapies leads to suboptimal health outcomes, lower quality of life, and increased mortality and health care costs. Adherence among patients with chronic conditions is particularly low, dropping most dramatically after the first 6 months of therapy. ${ }^{2}$ In multiple sclerosis (MS), treatment discontinuation is common, especially during the first months of therapy, increasing the risk of relapse, disease progression, and hospitalization. Disease-modifying therapies (DMTs) aim to reduce the frequency and severity of MS relapses and slow disease progression. ${ }^{3}$ Despite the indubitable benefits, there are several factors associated with DMTs that can impact patient adherence, including inconvenient methods and schedules of administration, long periods of therapy, side effects, or lack of direct relief of recurrent MS-related symptoms. Only long-term adherence to recommended DMT regimens ensures full treatment benefits. Health care professionals play a key role in the management of MS, encouraging the patients to persist with their therapies
Department of Neurology and Neurorehab, AZ Alma, Campus Sijsele, 132 Gentse Steenweg, Sijsele,

Damme 8340, Belgium

Tel +325072805 I

$\mathrm{Fax}+3250728008$

Email danny.decoo@azalma.be 
and providing education on the treatment-adherence benefits. This is the first survey to provide an overview of treatment adherence among Belgian patients with MS, as perceived by their neurologist in clinical practice.

\section{Materials and methods}

This survey of Belgian neurologists treating patients with MS was conducted between June and July 2014. Neurologists with recognized clinical experience in MS were contacted by phone, informed of the purpose of the survey, and invited for a face-to-face interview at their workplace. All neurologists provided written agreement to participate in the survey. As no patient data is included in this market research survey, no ethical approval was requested. The survey was based on a semistructured questionnaire (in French or Dutch) comprising questions regarding the perception of the treatment-adherence level among MS patients, the use of specific terms (eg, "compliance", "adherence") when talking to patients about their treatment, methods of administration, use of MS nurses, and patients' knowledge of nonadherence consequences.

\section{Results}

\section{Survey participants}

A total of 41 Belgian neurologists participated in the survey. Of these, $50 \%$ worked in a hospital, $10 \%$ had a private practice, and $40 \%$ worked in both a hospital and a private practice. The mean duration of clinical practice was 19 years.

\section{Patient adherence in all treatment areas}

In the first part of the questionnaire, neurologists were asked what came to mind when "correct intake of medication" was mentioned. The majority (63\%) indicated specific words, such as "compliance" (27\%) or "adherence" (17\%). A definition of adherence was mentioned by $27 \%$ of neurologists, the most common being "taking or administering the treatment correctly" (15\%) and "taking medication as the prescriber suggests" (5\%) (Table 1).

The majority of neurologists $(88 \%)$ indicated that frequent discussions with patients about treatment adherence were important for the following main reasons: correct treatment administration is crucial for efficacy (61\%), frequent discussions can help in finding causes of poor compliance (17\%), and determining true compliance (ie, adherence can be overestimated, as patients often do not report noncompliance to their doctors) (11\%).

The main tools/ways of improving treatment adherence were: "patient's contact with professionals" (61\%) and "practical aids" (56\%), such as alert systems (SMS,
Table I Neurologists' perception of main issues related to correct treatment administration and words most commonly used to describe treatment administration according to the medical prescription $(n=4 I)$

\begin{tabular}{ll}
\hline Category & Percentage \\
\hline Use of specific wording & 63 \\
Compliance & 27 \\
Adherence & 17 \\
Compliant with the medication dosing & 12 \\
Therapeutic compliance & 5 \\
Definition & 27 \\
Taking/administering the treatment correctly & 15 \\
Taking medication as the prescriber suggests & 5 \\
Reasons for incorrect administration & a \\
Patient noncompliance & 12 \\
Impact on efficacy & 7 \\
Importance of physician-patient communication & 7 \\
\hline
\end{tabular}

Note: ancluding high dosage frequency, side effects, excessively frequent doses, poor patient motivation.

electronic devices, phone), pillboxes, calendars, diaries, treatment timetables, medication cards, and information brochures.

\section{Patient adherence in MS}

More than half of neurologists (56\%) perceived the current treatment-adherence level among MS patients as good, 32\% as average, and $12 \%$ as excellent. Physicians who described the current adherence level as "good" or "excellent" provided the following justifications for their choices: physicians' and patients' improved knowledge of the disease and available treatments (36\%), patients' fear of disease progression or relapse in cases of nonadherence $(36 \%)$, use of selfinjectors (29\%), and observation based on clinical practice (18\%). Those who described adherence level as "average" indicated side effects, need for daily injections, incorrect understanding of the disease, or forgetfulness (23\% each) as justifications.

Approximately half of respondents (49\%) defined good treatment adherence in MS based on the "percentage of medication doses taken correctly" (Table 2). The majority of neurologists $(64 \%)$ indicated intolerance (including side effects, skin toxicity, painful injections, flu-like syndrome) as the main cause, and all indicated insufficient efficacy as the main consequence of poor adherence in MS (Table 3).

In the opinion of $34 \%$ of neurologists, poor tolerance might also be a consequence of poor adherence; of those, $64 \%$ indicated increased risk of adverse events in cases of poor adherence as a justification. For $17 \%$ of respondents, there was no link between adherence and tolerance. The remaining $49 \%$ expressed different opinions, of whom $85 \%$ considered 
Table 2 Definition of good treatment adherence in multiple sclerosis $(n=4 I)$

\begin{tabular}{ll}
\hline & Percentage \\
\hline Percentage of patients taking their medication correctly & 49 \\
$95 \%$ & 7 \\
$>90 \%$ & 7 \\
$90 \%$ & 7 \\
$85 \%-90 \%$ & 5 \\
$>80 \%$ & 15 \\
Taking medication according to physician's prescription & 44 \\
Good clinical outcome $^{\mathrm{a}}$ & 7 \\
Manageable disease $^{2}$ & 5 \\
\hline
\end{tabular}

Note: ${ }^{\text {BBased }}$ on the results of magnetic resonance imaging, regular blood tests, and follow-up.

poor tolerance as the cause and not the consequence of poor adherence.

The majority of neurologists (73\%) thought that MS patients were generally aware of the possible consequences of incorrect treatment administration. Of those who had the opposite opinion, $82 \%$ indicated that patients were not always aware of the lack of treatment efficacy following nonadherence.

About half of respondents indicated patients with lower intellectual capacity or from lower socio-economic classes as more likely to have poor adherence behavior (Table 4). In addition, $39 \%$ of neurologists indicated that

Table 3 Causes and consequences of poor adherence in multiple sclerosis $(n=4 I)$

\begin{tabular}{ll}
\hline & Percentage \\
\hline Causes & 64 \\
Intolerance & 31 \\
Method of administration & 31 \\
Forgetfulness & 26 \\
Psychological problems/cognitive impairment/ & \\
demotivation/depression & 26 \\
Lack of disease knowledge & 18 \\
Feeling well & 13 \\
Specific occasions/periods & \\
Frequency of injections & 10 \\
Disease progression & 8 \\
Doubt about the importance of treatment & 8 \\
Consequences & \\
Insufficient efficacy & 100 \\
Insufficient efficacy, new relapses & 71 \\
Disease progression & 42 \\
Not treated as prescribed: no response & 12 \\
Aggravation of disability & 10 \\
Recurrences & 7 \\
New lesions on magnetic resonance imaging & 5 \\
Switch to second-line therapy & 15 \\
Expensive and ineffective treatment & 5 \\
\hline Note: & \\
\hline
\end{tabular}

Note: aHolidays, festive season, shift work, etc.
Table 4 Patients likely to have poor adherence behavior $(n=4 I)$

\begin{tabular}{ll}
\hline & Percentage \\
\hline Lower intellectual capacity/underprivileged/ & 51 \\
lower social classes & \\
No support & 20 \\
Not aware of need for treatment & 20 \\
Psychological/cognitive problems & 17 \\
Forgetful & 14 \\
Older & 11 \\
Experiencing many side effects & 11 \\
Nonchalant & 11 \\
Not accepting the disease & 9 \\
Fear of injections & 9 \\
Taking many medications & 9 \\
\hline
\end{tabular}

adherence may vary during specific times of the year, such as holidays (100\%), festive seasons $(38 \%)$ or other busy periods $(19 \%)$.

\section{Adherence in clinical practice}

On a scale of $1-10$, with $1=$ "not very important" and $10=$ "very important", $44 \%$ of neurologists attributed a score of 10 to the importance of treatment adherence in their practice (mean score 9.0). The majority of respondents $(71 \%)$ justified their score by indicating treatment adherence as the key to successful treatment. Among the key aspects regarding treatment adherence raised at the initial visit, $58 \%$ of neurologists indicated the importance of correct treatment administration, and $45 \%$ the aims of therapy (Table 5). The mean time spent on discussing treatment adherence during the initial consultation was 11 minutes: $24 \%$ of doctors spent 5 minutes, $24 \%$ spent 10 minutes, $16 \%$ spent 15 minutes, $16 \%$ spent $>15$ minutes, $13 \%$ spent $6-9$ minutes, and $8 \%$ spent $<5$ minutes discussing this issue. Among the neurologists who indicated the level of treatment adherence in MS as "good" or "excellent" $(68 \% ; n=28), 39 \%$ indicated they

Table 5 Key aspects concerning treatment adherence discussed with multiple sclerosis patients at treatment initiation $(n=4 I)$

\begin{tabular}{ll}
\hline & Percentage \\
\hline Need for correct treatment administration/compliance & 58 \\
Aims & 45 \\
Fewer side effects & $\mathrm{II}$ \\
Disease stabilization & $\mathrm{II}$ \\
Slowdown of disease progression & 8 \\
Improvement of quality of life & 8 \\
Answering questions & 13 \\
Importance of achieving the aims & 13 \\
Explaining the treatment & 8 \\
Cost of treatment & 5 \\
\hline
\end{tabular}


spent $<10$ minutes, $36 \%$ spent $10-15$ minutes, and $18 \%$ spent $\geq 20$ minutes discussing treatment adherence with their patients at the initial visit (7\%; number not indicated). Among those who described the level of treatment adherence in MS as "average" (31\%; $n=13), 46 \%$ indicated they spent $<10$ minutes, $39 \%$ spent $10-15$ minutes, and $8 \%$ spent $\geq 20$ minutes discussing this issue ( $8 \%$; number not indicated).

Almost half of respondents (44\%) indicated they had already dealt with patients who had failed to initiate the prescribed treatment. These physicians indicated patients who doubted or did not trust the treatment efficacy (72\%), patients with anxiety problems $(39 \%)$, or patients who preferred alternative practitioners $(11 \%)$ as those who most often failed to initiate their treatment.

\section{Ways to improve adherence}

Neurologists indicated family members or other supporting persons $(76 \%)$, neurologists $(59 \%)$, MS nurses $(49 \%)$, and general practitioners $(46 \%)$ as those who, in addition to patients themselves, could influence patients' treatment adherence.
The main tools or supports used to help the patients achieving good adherence were: MS nurses (46\%), discussion with or motivating the patient (28\%), and regular follow-up visits (21\%). In particular, respondents indicated MS nurses $(36 \%)$, initial information, explanation, or brochures $(28 \%)$, or frequent visits/planned follow-up visits (23\%) as the main means that could be offered to patients during the initial 3-6 months of treatment. The majority of doctors $(63 \%)$ indicated specific alert systems, such as reminders via text messages or smartphone applications, as potential tools or supports that could be developed to improve treatment adherence (Table $\mathrm{S} 1$ ).

On a scale of $1-10$, with $1=$ "cannot improve adherence" and $10=$ "can improve adherence", "encouraging the patients to take their medication correctly" was indicated as the most important factor influencing the treatment-adherence level among MS patients (score 9.1) (Figure 1).

\section{Discussion}

Compliance with treatment strategies is a key factor toward successful outcomes in disease management. While "compliance" refers strictly to the need for a

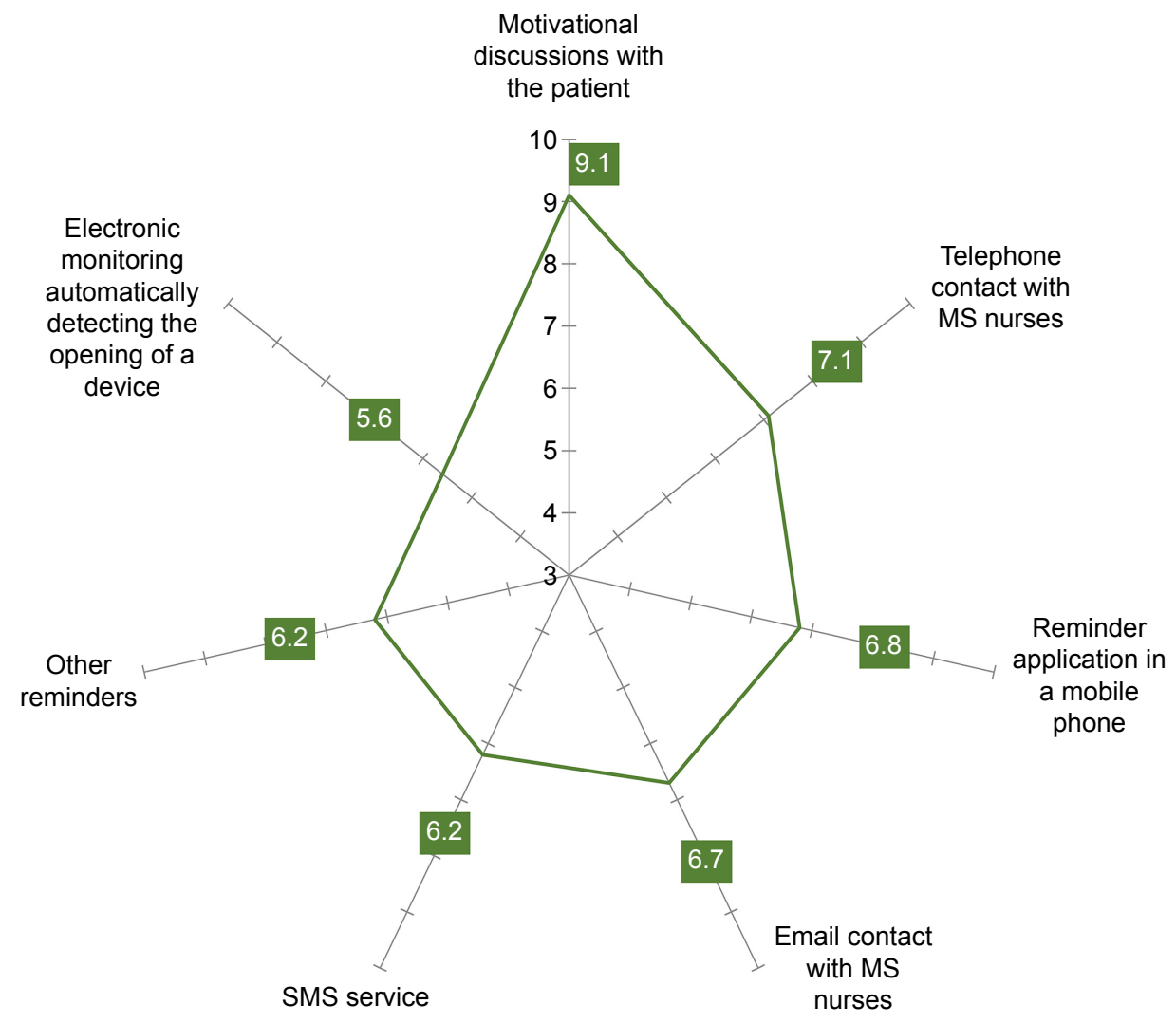

Figure I Level of motivational value of potential supports/tools improving patient adherence to multiple sclerosis (MS) treatment.

Notes: Neurologists assessed the extent to which potential tools/supports can improve treatment adherence on a scale of I-I0, where I= "cannot improve adherence" and $10=$ "can improve adherence". 
patient to follow instructions, in the concept of "treatment adherence", the patient plays an active role, recognizing the need for medication, persisting with the therapy, and being compliant. The definitions of "compliance" and "adherence" are often misunderstood or are used interchangeably. It is thus the role of physicians to provide explanations and highlight the importance of treatment adherence to their patients.

Several previous studies based on retrospective cohort designs or from a patient perspective have assessed treatment adherence in $\mathrm{MS},{ }^{4-8}$ and a few published physician questionnaire-based studies have reported neurologists' choices and experiences with individual DMTs. ${ }^{9-11}$ However, little is known about neurologists' views on patient adherence in MS. This survey reports Belgian neurologists' perceptions of treatment adherence and factors affecting adherence among MS patients.

In our survey, the vast majority of neurologists indicated treatment adherence as a key factor toward an effective treatment. Therefore, the focus on treatment adherence along the patient's treatment course seems increasingly to become a routine in the neurologists' clinical practice. The majority of respondents used the terms "adherence" or "compliance" when talking about treatment adherence. Although only $15 \%$ of physicians defined "adherence" as taking/administering medication correctly, about half of respondents understood "compliance" as taking medication according to medical prescription. About $25 \%$ of respondents considered that treatment adherence was a patient's responsibility, and generally spent only about 5 minutes discussing treatment adherence with their patients during the initial visit. These numbers suggest that although most physicians are aware of the importance of treatment adherence in MS management, there is a certain variability in the specific wording and definitions used by neurologists, indicating a need for clear guidelines on treatment adherence. In addition, physicians who considered the level of treatment adherence as "good" or "excellent" were keener to spend more time on adherence counseling than those who described treatment adherence as "average". However, due to the small number of physicians included in this survey, a direct correlation between reported adherence and time spent on counseling could not be established.

More than half of neurologists described the treatmentadherence level among Belgian MS patients as "good" or "excellent". Similarly, in a previous international survey of 280 neurologists from Australia, Canada, France, Germany, Italy, Spain, and the UK, more than half of respondents (59\%) indicated they had faced no compliance issues when treating MS patients. ${ }^{11}$ This suggests that treatment-adherence levels among MS patients are similar in developed countries. However, adherence rates might vary according to the definition of adherence used. In our survey, almost half of respondents defined adherence as the percentage of doses taken correctly (most commonly with a cutoff of $80 \%$ ). A similar adherence definition was used in a previous American survey of neurologists, ${ }^{10}$ whereas in the Global Adherence Project (GAP) study it was defined as "not missing a single DMT injection within 4 weeks before the study". ${ }^{9}$ Furthermore, adherence rates might also reflect cultural differences, as in some countries patients are more likely to adhere to their doctors' recommendations. ${ }^{12}$

In our survey, neurologists who perceived the adherence level of MS patients as "good" or "excellent" indicated improved patient and physician knowledge of the disease as resulting in better patient motivation, and patient awareness of disease progression in cases of nonadherence as justifications for their opinion. Nevertheless, for a considerable proportion of respondents (32\%), the adherence level in MS was "average". Of note, none of the respondents considered the adherence level as "poor". Consistent with previous reports, Belgian neurologists indicated side effects, need for daily injections, misunderstanding of adherence, and forgetfulness as the main factors affecting adherence. ${ }^{4,8,9,11,13,14}$ Furthermore, patient adherence might also vary at specific periods of the year, particularly holidays and other busy periods when a daily routine is generally interrupted. The importance of uninterrupted compliance should be highlighted when discussing adherence with patients.

For the majority of Belgian neurologists, treatment efficacy depends directly on the adherence level, and most neurologists believe that MS patients are aware of the consequences of their nonadherence, such as increased relapse rates, risk of disease progression and hospitalization, and subsequent increased use of health care resources. ${ }^{5,7,12}$

Patient sociodemographic status may also affect treatment adherence. In our survey, patients with lower intellectual capacity or from lower socioeconomic classes were indicated as more likely to have poor treatment adherence, which is consistent with previous reports. ${ }^{15,16}$ Furthermore, higher levels of nonadherence have also been reported more among depressed and despairing MS patients. ${ }^{8}$

Adherence rates in chronic diseases, including MS, have been shown to decrease over time, and MS patients who persist with therapy throughout the initial period are more likely to achieve long-term adherence. ${ }^{17}$ Therefore, patient education 
preceding therapy is crucial. In our survey, neurologists indicated compliance with treatment regimens and the aims of therapy as major issues discussed with patients at the initial visit. Almost half of neurologists spent 5-10 minutes and only $32 \%$ spent $\geq 15$ minutes discussing treatment adherence at the initial visit. The previous GAP study reported that physicians spending more time discussing treatment adherence generally had dealt with more adherent patients. ${ }^{9}$

Various tools and supports are currently available to help MS patients remain treatment-adherent. In this survey, almost half of neurologists indicated MS nurses as the most commonly offered support to MS patients, particularly during the first 3-6 months of treatment, when patients adapt to the medication and its way of administration and often deal with side effects. MS nurses play an active role in patients' treatment adherence, assisting them in their daily life and being directly involved in their education and decision making, thereby being a good source of practical information regarding treatment effectiveness or possible side effects, management of disease symptoms, and available support services. Patient motivation and good doctor-patient communication are important ways to improve treatment adherence. ${ }^{18,19}$ Patients should be educated about the nature of their disease, particularly the variable and unpredictable course of MS. Ongoing reinforcement of the value of treatment is essential to maintain treatment adherence. The role of motivational counseling by physicians or MS nurses in the improvement of patient adherence has previously been highlighted; such counseling, performed either face to face or via a telephone call, would focus on the reasons and ways to fight treatment discontinuation and how to cope with the side effects or fear of therapy. ${ }^{13,20}$

The majority of neurologists indicated specific alarm systems, set up in mobile phones, smartphones, or email inboxes, as potential tools that could improve treatment adherence among MS patients. Daily contact with a personal nurse or physician was also considered. The respondents also suggested a free telephone number or an "MS league" offering support and information regarding medication and side effects. In a previous patient-based survey, a prompt card with details of MS-support services available, which could be given to patients at the initial visit, was suggested to be a useful tool potentially improving treatment adherence. ${ }^{6}$

Potential limitations of this survey include the small number of participants and lack of prespecified treatment-adherence definition. Therefore, it is likely that the respondents' interpretation of some terms used either in the questionnaire or in the neurologists' answers might have varied. Furthermore, as this survey was conducted among French- and Dutch-speaking neurologists, regional differences in health care provision and terminology might be reflected in the results. Additionally, this survey did not evaluate neurologists' prescribing behaviors, which can also affect patient adherence.

At the time the survey was completed (July 2014), DMT options for Belgian MS patients were classified as first-line treatments (IFN $\beta 1 \mathrm{a}$, subcutaneous injection three times a week; IFN $\beta 1 \mathrm{~b}$, subcutaneous injection once every 2 days; IFN $\beta 1 \mathrm{a}$, intramuscular injection once a week; glatiramer acetate, subcutaneous injection once a day) and second-line treatments (natalizumab, intravenous injection once every 4 weeks, and fingolimod once a day). Two other oral treatments (teriflunomide and dimethyl fumarate) were also available under restrictive use of a medical need program.

In conclusion, in the view of neurologists participating in this survey, treatment adherence among Belgian MS patients is generally good, thanks to improved patient and physician education and knowledge of the disease and various strategies commonly used to improve adherence. Although the importance of treatment adherence is well understood, the adherence definitions used vary among Belgian neurologists. Furthermore, the neurologists' perception of adherence level in MS seemed to be associated with longer counseling at the initial visit; however, due to the small number of survey participants, a direct correlation could not be established. Nevertheless, the present findings confirm that treatment adherence is a relevant and important issue in the clinical practice of neurologists, who are conscious of various obstacles to treatment adherence, the consequences of noncompliance, and the vital role they play in patient treatment adherence.

\section{Acknowledgments}

We thank Medistrat for their support in developing this survey. We also thank Urszula Miecielica (XPE Pharma and Science), who provided medical writing services on behalf of Biogen. This study was funded by Biogen.

\section{Disclosure}

MV has participated in several industry-sponsored clinical trials in the field of multiple sclerosis, sponsored by Biogen, Merck-Serono, Bayer Healthcare, GSK, and Novartis. He has received research support from Biogen, Merck-Serono, Novartis, Teva, and Bayer Healthcare, and travel expenses for attending meetings from Bayer Healthcare, Biogen, Novartis, Merck-Serono, Pfizer, and Sanofi-Aventis. He has also received personal compensation for consulting activities 
and participation in an advisory board from Bayer Healthcare, Novartis, and Biogen, and for lectures from Bayer Healthcare, Merck-Serono, and Biogen. DD has participated in several industry-sponsored clinical trials in the field of multiple sclerosis, sponsored by Biogen, Merck-Serono, Bayer Healthcare, Novartis, Hoffman-Roche, Sanofi-Genzyme, and Teva. He has also received travel expenses for attending meetings and compensation for consulting activities and advisory boards from the aforementioned companies.

\section{References}

1. Lugaresi A. Addressing the need for increased adherence to multiple sclerosis therapy: can delivery technology enhance patient motivation? Expert Opin Drug Deliv. 2009;6(9):995-1002.

2. Osterberg L, Blaschke T. Adherence to medication. N Engl J Med. 2005; 353(5):487-497.

3. Lim SY, Constantinescu CS. Current and future disease-modifying therapies in multiple sclerosis. Int J Clin Pract. 2010;64(5):637-650.

4. Di Battista G, Bertolotto A, Gasperini C, Ghezzi A, Maimone D, Solaro C. Multiple Sclerosis State of the Art (SMART): a qualitative and quantitative analysis of therapy's adherence, hospital reliability's perception, and services provided quality. Mult Scler Int. 2014;2014:752318.

5. Steinberg SC, Faris RJ, Chang CF, Chan A, Tankersley MA. Impact of adherence to interferons in the treatment of multiple sclerosis: a nonexperimental, retrospective, cohort study. Clin Drug Investig. 2010; 30(2):89-100

6. Syed M, Rog D, Parkes L, Shepherd GL. Patient expectations and experiences of multiple sclerosis interferon $\beta$-1a treatment: a longitudinal, observational study in routine UK clinical practice. Patient Prefer Adherence. 2014;8:247-255.

7. Tan H, Cai Q, Agarwal S, Stephenson JJ, Kamat S. Impact of adherence to disease-modifying therapies on clinical and economic outcomes among patients with multiple sclerosis. Adv Ther. 2011;28(1):51-61.

8. Treadaway K, Cutter G, Salter A, et al. Factors that influence adherence with disease-modifying therapy in MS. J Neurol. 2009;256(4): $568-576$.
9. Devonshire V, Lapierre Y, Macdonell R, et al. The Global Adherence Project (GAP): a multicenter observational study on adherence to disease-modifying therapies in patients with relapsing-remitting multiple sclerosis. Eur J Neurol. 2011;18(1):69-77.

10. Hanson KA, Agashivala N, Wyrwich KW, Raimundo K, Kim E, Brandes DW. Treatment selection and experience in multiple sclerosis: survey of neurologists. Patient Prefer Adherence. 2014;8:415-422.

11. Rinon A, Buch M, Holley D, Verdun E. The MS Choices Survey: findings of a study assessing physician and patient perspectives on living with and managing multiple sclerosis. Patient Prefer Adherence. 2011;5:629-643.

12. Lugaresi A, Rottoli MR, Patti F. Fostering adherence to injectable disease-modifying therapies in multiple sclerosis. Expert Rev Neurother. 2014;14(9):1029-1042.

13. Caon C, Saunders C, Smrtka J, et al. Introduction: adherence to diseasemodifying therapies - key to optimizing outcomes in relapsing multiple sclerosis. J Neurosci Nurs. 2010;42(5 Suppl):S1-S4.

14. Patti F. Optimizing the benefit of multiple sclerosis therapy: the importance of treatment adherence. Patient Prefer Adherence. 2010;4:1-9.

15. Hadjigeorgiou G, Dardiotis E, Tsivgoulis G, et al. Observational study assessing demographic, economic and clinical factors associated with access and utilization of health care services of patients with multiple sclerosis under treatment with interferon beta-1b (EXTAVIA). PLoS One. 2014;9(11):e113933.

16. Portaccio E, Amato MP. Improving compliance with interferon- $\beta$ therapy in patients with multiple sclerosis. CNS Drugs. 2009;23(6):453-462.

17. Saini SD, Schoenfeld P, Kaulback K, Dubinsky MC. Effect of medication dosing frequency on adherence in chronic diseases. Am J Manag Care. 2009;15(6):e22-e33.

18. Ha JF, Longnecker N. Doctor-patient communication: a review. Ochsner J. 2010;10(1):38-43.

19. Zolnierek KB, Dimatteo MR. Physician communication and patient adherence to treatment: a meta-analysis. Med Care. 2009;47(8): 826-834.

20. Smrtka J, Caon C, Saunders C, Becker BL, Baxter N. Enhancing adherence through education. J Neurosci Nurs. 2010;42(5 Suppl): S19-S29. 


\section{Supplementary material}

Table SI Potential supports and tools improving adherence in multiple sclerosis (MS); $(n=4 I)$

\begin{tabular}{ll}
\hline & Percentage \\
\hline Alarm systems & 63 \\
Reminder via SMS, smartphones & 44 \\
Smartphone applications & 22 \\
Daily contact with an MS nurse & 17 \\
Daily contact with a physician & 15 \\
Treatment timetables & 15 \\
Specific discussions with a physician & 12 \\
Calendar/diary & 10 \\
MS league $^{\text {a }}$ & 7 \\
Injection pens recording data & 7 \\
\hline
\end{tabular}

Note: ${ }^{a}$ Free telephone number available to MS patients.

\section{Publish your work in this journal}

Patient Preference and Adherence is an international, peer-reviewed, open access journal that focuses on the growing importance of patient preference and adherence throughout the therapeutic continuum. Patient satisfaction, acceptability, quality of life, compliance, persistence and their role in developing new therapeutic modalities and compounds to optimize clinical outcomes for existing disease states are major areas of interest for the journal. This journal has been accepted for indexing on PubMed Central The manuscript management system is completely online and includes a very quick and fair peer-review system, which is all easy to use. Visit http://www. dovepress.com/testimonials.php to read real quotes from published authors.

Submit your manuscript here: http://www.dovepress.com/patient-preference-and-adherence-journal 